\title{
Sensory analysis of traditional balsamic vinegars: current state and future perspectives
}

\author{
Federico Lemmetti, Lisa Solieri, Tommaso Bonciani, Gabriele Zanichelli, Paolo Giudici \\ UNIMORE Microbial Culture Collection, Department of Life Sciences, University of Modena \\ e Reggio Emilia, Italy
}

\begin{abstract}
Quality evaluation of traditional balsamic vinegar (TBV) is primarily based on sensory analysis. For every TBV batch, official sensory panels give a final score, which determines its assessment into quality and price categories. Therefore, an effective and objective sensory analysis is a core aspect in TBV production and marketing and it should fulfill at least two conditions: i) the panelists have been properly trained on the TBV features; ii) the panelists have to be free from any psychological and physical conditions which might affect human judgments. Traditionally, a panel of trained members assesses the TBV sensory attributes evaluating visual, olfactory, gustatory and trigeminal features at the same time. The result is that visual appearance significantly affects the subsequent stages of the sensory analysis, and even the olfactory and gustatory sensations will greatly affect each other. The aim of this work was to review the procedures for the sensory analysis of TBV and to define a set of TBV attributes. A new assessment questionnaire has been proposed to establish the appropriate sensory vocabulary for a complete description of TBV sensory properties.
\end{abstract}

\footnotetext{
Correspondence: Paolo Giudici, Department of Life Sciences, via Giovanni Amendola 2, 42122 Reggio Emilia, Italy.

Tel.: +39.0522522034 - Fax: +39.0522522027.

E-mail: paolo.giudici@unimore.it

Key words: questionnaire, interval scale, independence judgment, balsamic vinegar, sensory analysis.

Contributions: PG and FL conceived the review and the assessment questionnaire; TM and GZ collected and analyzed the references; LS wrote and discussed the manuscript.
}

Conflict of interests: the authors declare no conflict of interests.

Received for publication: 26 July 2014.

Revision received: 18 August 2014.

Accepted for publication: 22 August 2014.

This work is licensed under a Creative Commons Attribution 3.0 License (by-nc 3.0).

(C) Copyright F. Lemmetti et al., 2014

Licensee PAGEPress, Italy

Acetic Acid Bacteria 2014; 3:4619

doi:10.4081/aab.2014.4619

\section{Introduction}

Traditional balsamic vinegars (TBVs) of Modena (TBVMO) and Reggio Emilia (TBVRE) are two condiments with the protected denomination of origin (PD0) status, which are produced in the Italian districts of Modena and Reggio Emilia, respectively. Technological and microbiological aspects in TBV production have been extensively discussed in recent papers. ${ }^{1-3}$ The production procedures are coded by the rules of the PDOs, ${ }^{4,5}$ which include the sensory evaluation by an official panel as the final step. The sensory analysis is compulsory to get the PDO and the score achieved determine the quality ranking of the TBV batch. In particular, according to extant assessment procedures operated by the Consortia (associations of producers) TBVM0 samples can be of two quality and price levels (affinato and extravecchio), whereas TBVRE samples have three quality levels (aragosta, argento and oro). As sensory properties are related to price, evaluation of TBVMO and TBVRE must be objective. To date, the official sensory procedures are very similar for the two PD0 vinegars and they include the evaluation of visual descriptors, followed by olfactory perception and tasting. For TBVRE the panelists must assign a score to any of the following descriptors: viscosity, color and clearness for the visual features; delicacy, defectiveness, persistence and acidity for olfactory features; fullness, maturity, harmony and acidity for gustatory features. The panel is composed of five members from the official list of ABTRE expert tasters registered in the Chamber of Commerce of Reggio Emilia. They individually taste and express their evaluation filling a structured questionnaire: each descriptor has a pre-printed ranking of scores and the panelist has to sign which match better his evaluation. Structured questionnaire with pre-printed categorical scale force the choice of the panelists, limit their independence of judgment and increase the end effect ${ }^{6-8}$

\section{Limits of the current tasting procedures}

Food perception and preference rely on the ability to combine information from both the taste and olfactory systems. ${ }^{9}$ For sensory evaluation the concept of independent judgments is not realistic in a literal sense, because all individuals participating in tests have experience as product users and their responses reflect this dependence ${ }^{10}$ Food tasting presents very tricky obstacles, hidden or difficult to identify, since the visual appearance significantly affects the results of the subsequent stages of the sensory analysis, and even the olfactory and gustatory sensations will greatly affect each other (halo effect). From a sensory design standpoint, the use of a balanced serving order and other design considerations enable to minimize the impact of this dependence, mainly whether it is visual, olfactory, gustatory and trigeminal. ${ }^{11-}$

${ }^{13}$ In the case of TBV sensory analysis, the tasting procedure consists 
of a sequence of tests that could alter significantly the final sensory score and the panelist preferences, because the visual appearance assessed at the first step could strongly affect the subsequent sensorial perceptions irrespectively to the real or potential sensory perception. Previous studies on TBVRE showed that the final preference scores were significantly related with the appearance of the samples and, mainly, with their chemical and physical composition. This relationship is based on the common practice to inform panelists about the Brix and acidity values of the samples they are tasting, with the declared mean to help the panel in the sensory analysis. The assumption is that the information of Brix and acidity values facilitates the proper sensory evaluation. However, several evidences showed that this information compromises completely the analysis, because the member of the panel force their judgment irrespectively to what they feel. ${ }^{14}$

Furthermore, a previous work, ${ }^{15}$ showed that the scores assigned to the individual TBV descriptors are related to each other because the panelists, once identified vinegar of their preference from any of the point of view of taste, visual or olfactory, would reward it for any other sensory attributes regardless of their individual judgment. To try to clarify this result, seven well-trained panelists have been interviewed about their personal approach to the sensory evaluation of TBV. Their common behavior was the following: after preliminary visual and olfactory analyses, mainly addressed to identify serious defects, they proceed to taste and then to mentally decide the global score of the sample. Finally, the panelists fill in the questionnaire in its entirety and mark the scores for each descriptor to get the global score previously decided. As a result, there is a high correlation among the sensory descriptors. All these evidences suggest that the sensory analysis currently used to evaluate TBV attributes, could be inefficient in maximizing the independence of judgment.

\section{The balsamic lexicon}

In sensory evaluation the terminology is a set of labels that the panelists agreed to be used to fully communicate their description of the sensory properties of the products being evaluated. The taste and flavor of foods and beverages need clear and unequivocal lexicons used with the same meaning by all the panelists; the definitions and references are also employed to further illustrate the product's characteristics or attributes. The purpose of a lexicon is four-fold: i) to collect a product frame of reference; ii) to generate the terms; iii) to review references and examples; iv) to develop a final attribute list. Numerous lexicons have been developed for a variety of products and procedures, i.e. for peanut flavor, ${ }^{16}$ hand feel properties, ${ }^{17}$ cheddar cheese, ${ }^{18}$ soymilk, ${ }^{19}$ honey ${ }^{20}$ wine. ${ }^{21}$ In the case of TBV, the first lexicon was developed by Giudici et al. ${ }^{14}$ with a group of 60 panelists and established 24 sensory attributes of traditional balsamic vinegar, referring to four macro-categories (appearance, aroma, taste and trigeminal sensations). The aroma includes the highest number of attributes (such as caramel, cooked must, dried prune, honey, apple, licorice, vanilla, mustard, carob, spices, coffee and chocolate). These attributes were frequently recognized by all the panelists in the majority of the samples examined. Other frequently used descriptors are related to the four consolidated tastes (sour, sweet, bitter, salty), the tactile perception of viscosity and the three trigeminal sensations of pungency, spiciness and astringency. Finally, the current descriptors for TBV have been demonstrated to be redundant and very far from the common sensations of the panelists and consequently difficult to be quantified and used. The lexicon was further expanded by Zeppa et al. ${ }^{22}$ with a set of 17 and 3 descriptors for aroma and taste, respectively.

\section{Toward a novel questionnaire for the balsamic vinegar sensory analysis}

A standard method of sensory evaluation offers many advantages, as can be seen in other products where it is well established, e.g., cheese,${ }^{23}$ wine,${ }^{24,25}$ and dairy products ${ }^{26}$ A systematic approach to sensory evaluation of olive oil has been described and regulated (EEC Reg. 2568/91). ${ }^{27-30}$ To date, a standard method for sensory analysis of vinegars does not exist. Sensory analysis has been used to discriminate vinegar samples on the basis of the raw material, ${ }^{31,32}$ or the elaboration method. ${ }^{33,34}$ Another work deals with the evaluation of effect of tasting protocol on the panelist performance. ${ }^{35}$

The design of a good assessment questionnaire for the sensory analysis cannot ignore the use of clear, unequivocal terms used with the same meaning by all the panelists, while it remains under the panelist's choice to determine the level of each attribute as being insufficient, optimal or too high. Furthermore, the assessment form should be easy-to-use, leaving the panelists concentrated on their sensory perceptions and not worried about the filling of the questionnaire. Finally, the structure of the questionnaire must not affect the expression of the sensory judgment, with numbers or other indications, in order to encourage the use of the whole width of the scale of sensory attributes. Taking into account the aforementioned requirements, a new questionnaire has been designed, divided into three sections (Figure 1), each of them related to one of the three sensory groups (olfactory, gustatory-olfactory and visual), and having its respective attributes. The chosen attributes were consistent with a one-dimensional concept, removing ambiguity and thus ensuring that all the panelists refer to the same sensory concept. ${ }^{36-38}$ The amplitude of each attribute is expressed in an interval scale by an unstructured 7 or $14 \mathrm{~cm}$ straight line, on which the panelist affixes a sign to express the degree of perception. The shorter lines are unipolar scales, with the maximum score being on the right edge, whereas the longer lines are bipolar scale and the maximum score is on the middle. ${ }^{39}$ Furthermore, we have inserted two or three verbal labels for the left, middle and right bounds of judgment.

Finally, the score is assigned to the corresponding lines after the sensory analysis by overlapping a semi-transparent mask, which presents the different numerical values for each descriptor (Figure 2). In the future the questionnaire could be digitally acquired and the data collected with not yet developed software. Like the previous evaluation form for TBVRE sensory analysis, the maximum score is 400 but there is no precise correspondence about the scores between the new and the old assessment forms.

\section{Tasting procedure}

During the procedure of tasting, maximum independence of judgment should be promoted, i.e. each panelist must feel completely free to express his/her sensory preferences without the pressure of being judged in his/her turn for the task that is being performed. A panelist influenced by the fear of making mistakes tends to avoid extreme judgments, positive and/or negative, and to stick to average values, making the responses totally useless. ${ }^{8,40}$ For this reason the individual judgments must remain anonymous to the rest of the panel. Furthermore, the panelists must be instructed on how to proceed with the sensory analysis; in particular, they must take responsibility for their own views on single attribute in a totally independent way. In this regard, it is very useful to carry out the olfactory analysis in a completely obscured vial/bottle and fill out the questionnaire with no further possibility of correction, and close the testing olfactory session before the visual assessment so that the interference between the individual sensory 
attributes is considerably limited. The tasting procedure must also define other factors, such as the temperature and the amount of sample to be tested, the specific gestures to follow for each sensory test, and finally the maximum number of samples to assess in each session.

The panelists may be requested to express a purely hedonistic comment or to recognize the specific level of certain qualitative attributes in relation to a reference standard. In the first case, the panel members do not require a specific preparation because their skills depend on the degree of sensitivity of his/her sense organs and on the ability to discern different samples based on describable traits. In the second case, training of assessors is mandatory according to the ISO guidelines, ${ }^{41,42}$ to assure the constancy of panelist's performance and the analytical judgment based on a quality scale defined by assessable standards.

Apart from the visual examination, during tasting the senses involved are the smell, the taste and the trigeminal system working all together to determine a global impression of taste, which is then decomposed to form the individual parts of the perception. Hybridization, synergy and suppression of individual molecules against the others can commonly occur. ${ }^{43,44}$ Moreover, according to the Helson's principle, the sensory effect of a gustatory stimulus of equal extent varies according to the laws of habituation, sensitization and contrast with the level of previous stimulation..$^{45}$ It is clear that the ability to decompose a complex experience in others less complex, is a basic requirement for a good panelist, but it must be supported by a simple, fast and objective tool of evaluation.

Therefore, to facilitate the isolation of each sensory character and reduce the mutual influence of each character on the next sensation, the new questionnaire has been designed to follow the natural sequence of sensory perceptions, such as: smell, tasting and visual inspection, respectively.

\section{Olfactory perceptions}

Olfactory sensations are the most difficult to ascertain because the words that can be used to define a large number of flavors is limited. Moreover, other well-known factors, such as antagonism, synergy, pseudo-domain, suppression and adaptation, make it very difficult to identify the aromas in a mixture. In addition, the sensory analysis could be complicated by the use of a specific scale for each flavor. In the specific case of TBV, the aroma recognition is difficult due to the complexity of the mixture and the aging, which tends to degrade the aromatic substances most frequently recognized in not aged food. Nevertheless, the flavor of the balsamic must play a key role in the

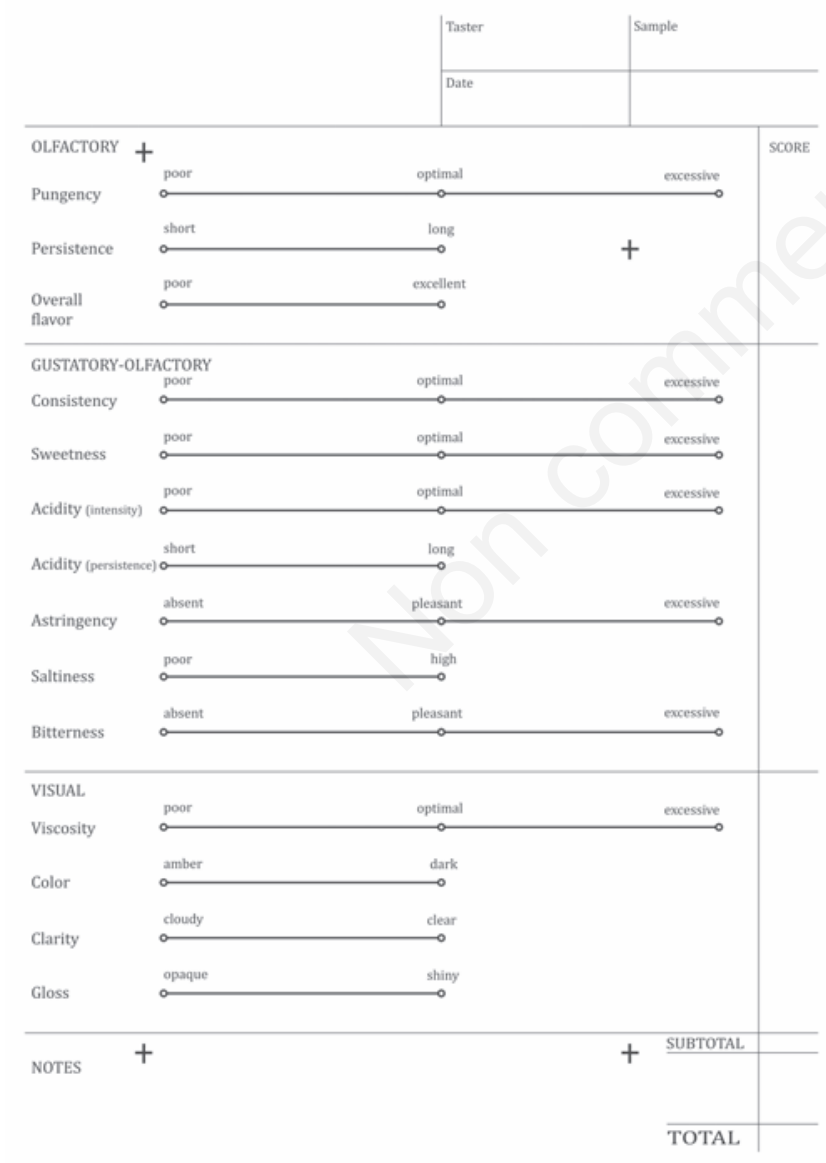

Figure 1. The proposed questionnaire for the assessment of balsamic vinegars. For each descriptor on the left, a non-numerical interval scale is represented as a continuous line, unipolar or bipolar.

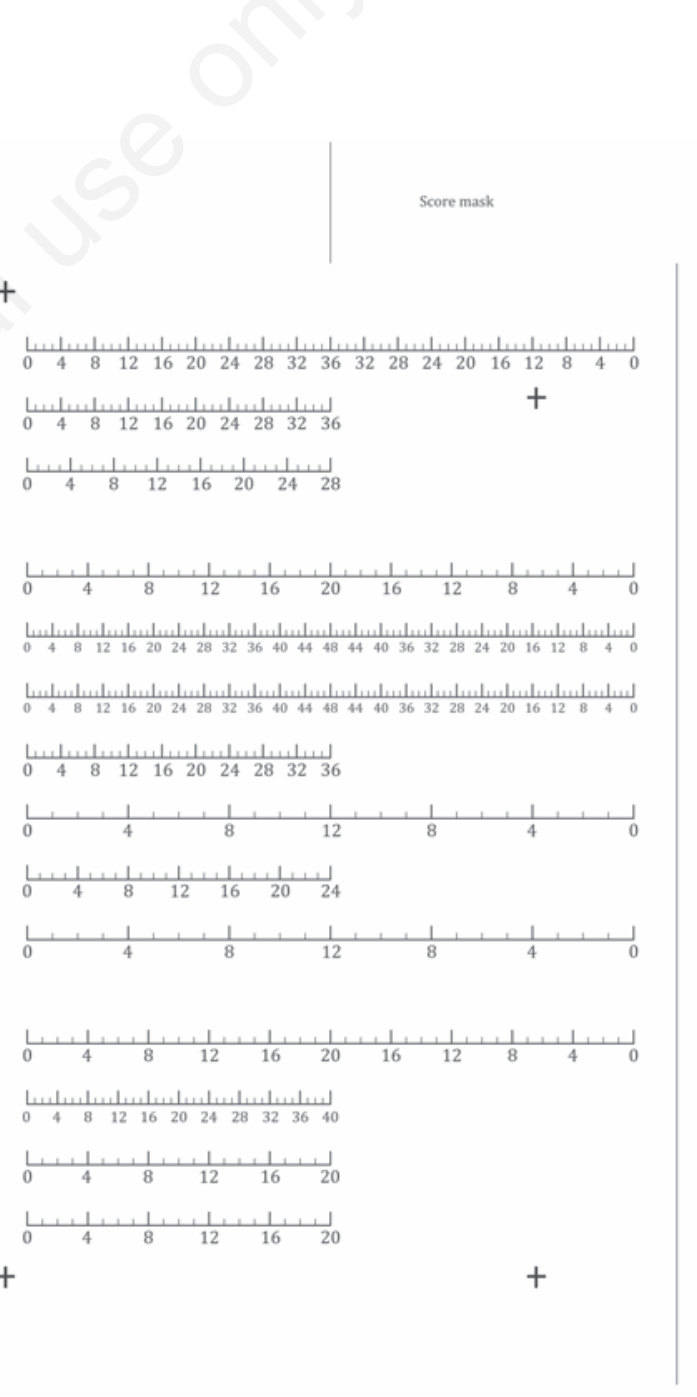

Figure 2. The sheet mask to be overlapped to the questionnaire for the computation of the score. 
assessment and, thus, it has been included as overall flavor descriptor which encompasses agreeableness, depth and complexity attributes.

\section{Gustatory perceptions}

The taste is the rawest feeling or rather the less refined. It let us make a first scan of the flavors of food (acidity, sweetness, bitterness, saltiness, neglecting the umami and metallic). In order to generate the flavor, the taste is complemented by tactile, thermal, olfactory and trigeminal perceptions. ${ }^{46}$ The flavor is always derived from a combination of the four basic descriptors (salty, sweet, sour and bitter) caused by soluble substances in the saliva. Even a single chemical substance can have multiple flavors at the same time. For example, the salts of sodium and lithium are typically salty; those of potassium are salty and bitter. Similarly, the organic acids differently affect the final taste of TBV. Whereas the tartaric acid is strong and dry, the succinic acid is weak and provides bitter and salty notes. Similarly, the lactic acid is moderately acid. Otherwise the malic and the citric acids are astringent and freshly sour, respectively. In relation to metabolites produced by acetic acid bacteria, the acetic acid is intense and pungent, while the gluconic acid derived from D-glucose ${ }^{47}$ is fresh and sweet. The sodium salts of the same acids vary the effect on the salinity according to the length of the organic chain. Not all carbohydrates, even if called sugars have sweet taste. Some proteins are used as sweeteners, while the majority of peptides are bitter. All these compounds are present in TBV at different concentrations, ${ }^{3}$ and their balance determines the final taste. During tasting, the combination of different flavors is not perceived contemporarily. The four flavors have indeed different initial times and persistence in the mouth; in the first moments of tasting the sweet taste prevails over the others and remains in the mouth about until swallowing (a few seconds); after a few seconds, there is the gradual reduction of the sweet flavors and an increase of sour and salty flavors, to end up with the acid and especially bitter aftertastes. To facilitate the work of decoding the panelist's feelings, the questionnaire considers the intensity of the four flavors, keeping the same sequence of the tasting sensory responses. Moreover, the assessment form includes the evaluation of the acidity persistence, which presumably lasts for the longest time after swallowing.

Another parameter affecting the sensory perceptions is the temperature of the sample. The cold enhances the freshness of acidity, dryness of bitterness and astringency, while it mitigates the sense of softness by the sugars (and other substances which give sensations of heat as the alcohols) and greasiness of substances such as glycerol. In contrast, the heat makes acidity and astringency less noticeable and enhances the sweetness and texture. The sensory characteristics of the four tastes are also related to different reactions by the oral mucosa. The sweet increases the secretion of thick and viscous saliva, whereas the acidity and saltiness an abundant and flowing one. Bitterness usually results in a loss of salivation because it is often combined with the sensation of astringency, not arising from the taste buds but from other receptors present on the mucous membranes of the mouth.

\section{Physical perceptions}

The tactile trigeminal sensations stem from the excitement of mechanical and chemical receptors connected to the trigeminal nerve, which is a sensory apparatus distinct from the others. We can therefore divide perceptions into physical (tactile in the strict sense of the word) and chemical. The first category includes the sensations related to consistency, viscosity, grittiness, oiliness and texture, in addition to the perception of the actual temperature of the sample. In the second category we can place sensations related to pain, discomfort and irritation such as astringency, pungency, spiciness, sizzling, metallic, pseudoheat and pseudo-freshness. ${ }^{48}$ For TBV, the most relevant attributes of this family are definitely pungency, viscosity (consistency) and astringency.

\section{Visual appearance}

Visual perceptions are very important elements of the judgment and should be done at the end of the evaluation procedure to avoid influence on the other analysis. The color of TBV ranges from amber to dark brown and it is usually associated to the age of the vinegar. ${ }^{49}$ Other visual attributes are brilliance and clearness. Brilliance is referred to the surface glossy aspect of a vinegar drop and it is the capacity to reflect the light creating the effect of lucidity, similar to a polished metal surface or a drop of mercury but transparent. Many pictures of commercial balsamic vinegars play on the brilliance, as for example, when they show a drop of balsamic that falls. Clearness is an attribute referred to its transparency. It is assessed by observing a thin layer of TBV through a transparent surface such as glass. The amount of matter in suspension and the size of the particulates may affect the clearness, making the vinegar opalescent or turbid, depending on the size of the particulates. Finally, the viscosity, also perceived during the gustatory test, refers to the thickness and the persistence of the layer of sample sliding along the walls of the flask.

\section{Guidelines for sensory score ranking}

For each sensory test some guidelines for its implementation have been given below, as well as the description of the characters and the corresponding maximum scores, expressed as a percentage of the whole evaluation. For a quick and concise reference of definitions of the terms commonly in use in sensory analysis see Appendix.

The smell test is performed without having observed the sample, that is, while the examination is being held, the vinegar is kept in a darkened flask. The three descriptors, accounting up to $25 \%$ of the total score, are: the pungency (9\%), the persistence (9\%) and the overall olfactory flavors (7\%). The last is a judgment of pleasantness that takes into account the presence of more or less pronounced characteristic aromas of the TBV. The pungency is the first example of a pyramidal (bipolar) scale descriptor: the score ranges from zero (poor) to 36 (optimal) and then decreases again to zero (excessive).

After the olfactory test, the gustatory-olfactory test is performed keeping the sample flask still obscured. The partial score is up to $50 \%$ of the total score, and the descriptors are: consistency (5\%), which expresses the tactile effect in the mouth mainly due to the density and viscosity; the sweetness (12\%), the intensity of the acidity (12\%), the persistence of the acidity (9\%), astringency (3\%), saltiness (6\%) and bitterness (3\%). With the exception of the persistence and the saltiness, which obviously cannot be excessive, all the descriptors have pyramidal scale like the pungency.

The visual test score can be worth up to $25 \%$ of the total score. In this test the taster evaluates: the viscosity (5\%), that is the ability of the liquid to adhere to the glass of the flask; the color (10\%) and the presence of undesirable reflections; the clearness (5\%), referred to the transparency of the sample; the gloss (5\%), i.e. the ability to reflect the light. The viscosity is the only visual test evaluated on a pyramidal scale, to penalize too viscous or too fluid samples.

\section{Conclusions}

The sensory analysis is a very powerful and useful tool, which, in addition to chemical and instrumental analyses, significantly contributes to the TBV quality assessment.

An essential prerequisite to ensure the effectiveness and reliability of the TBV sensory analysis entails with procedures that maximize the independence of judgment of the panelists. Accurate tasting proce- 
dures and clear evaluation questionnaires both contribute to assure good level of independence in sensory evaluation. The evaluation form suggested here has an easy-to-use layout which differs from those currently used by the Consortia of TBVMO and TBVRE, but it fulfills the most recent directives made on a scientific basis with regard to the sensory analysis. It includes clear, easily identifiable and officially recognized terms (i.e. bitter, sweet, salty, astringent and pungent) that have a clear meaning shared by all the panel members. Furthermore, the questionnaire lacks of categorical values to facilitate the use of the entire scale. Thanks to this simple layout, panelists can concentrate their attention more on the perceptions rather than on the compilation of the questionnaire; in the future the validation of this and other standardized sensory methods will overcome the drawbacks in the current TBV evaluation system, leading to a reliable and rationale classification of TBV samples into quality and price categories.

\section{References}

1. Giudici P, Gullo M, Solieri L, Falcone PM. Technological and microbiological aspects of traditional balsamic vinegar and their influence on quality and sensorial properties. Adv Food Nutr Res 2009;58:137-82.

2. Giudici P, Gullo M, Solieri L. Traditional balsamic vinegar. In: Solieri L, Giudici P, eds. Vinegars of the World. Berlin: SpringerVerlag; 2009. pp 157-77.

3. Solieri L, Gullo M, Giudici P. Traditional balsamic vinegar: a microbiological overview. In: Hui YH, Özgül Evranuz E, eds. Handbook of plant-based fermented food and beverage technology, 2nd ed. New York: CRC Press, Taylor and Francis Group; 2012. pp 609-30.

4. Ministero delle Politiche Agricole e Forestali. Provvedimento 15 maggio 2000, Disciplinare di produzione della denominazione di origine protetta "Aceto balsamico tradizionale di Modena". GU Ser. Gen. no. 124, 30/5/2000, pp 40-42.

5. Ministero delle Politiche Agricole e Forestali. Provvedimento 15 maggio 2000, Disciplinare di produzione della denominazione di origine protetta "Aceto balsamico tradizionale di Reggio Emilia". GU Ser. Gen. no. 124, 30/5/2000, pp 43-44.

6. Armstrong GA. Quantitative descriptive analysis (QDA) utilizing the human instrument, J Nutr Food Sci 1999;99:317-23.

7. Köster EP. Tonalité affective et maîtrise de la pollution odorante. In: Martin G, Laffort P, eds. Odeurs et désodorisation dans l'environnement, TEC et D0C. Paris: Lavoisier; 1991. pp 61-78.

8. Lawless $\mathrm{H}$, Malone G. The discriminative efficiency of common scaling methods. J Sens Stud 1986;1:85-98.

9. Verhagen JV, Engelen L. The neurocognitive bases of human multimodal food perception: sensory integration. Neurosci Biobehav Rev 2006;30:613-50.

10. Stone H, Bleibaum R, Thomas HA. Test strategy and the design of experiments. In: Stone H, Bleibaum R, Thomas HA, eds. Sensory evaluation practices, 4th ed. London: Academic Press; 2012. 11.

Stroebele N, De Castro JM. Effect of ambience on food intake and food choice. Nutrition 2004;20:821-38.

12. Auvray M, Spence C. The multisensory perception of flavor. Conscious Cogn 2008;17:1016-31.

13. Spence C, Shankar MU. The influence of auditory cues on the perception of, and responses to, food and drink. J Sensory Stud 2010;25:406-30.

14. Giudici P, Falcone PM, Scacco A, Lanza CM. Sensory analysis of the traditional balsamic vinegar. Industr Bevande 2009;224:27-42.

15. Lemmetti F, Falcone PM, Giudici P. Traditional balsamic vinegar: the role of the viscosity on the objective and perceived quality.
Industr Bevande 2013;244:9-21.

16. Johnsen PB, Civille GV, Vercellotti JR, et al. Development of a lexicon for the description of peanut flavor. J Sensory Stud 1987;3:9-17.

17. Civille GV, Dus CA. Evaluating tacticle properties of skin care products: a descriptive analysis technique. Cosmet Toiletries 1991;106:83-8.

18. Drake MA, Mcingvale SC, Gerard PD, et al. Development of a descriptive language for cheddar cheese. J Food Sci 2001;66:142227.

19. Day N'Kouka K, Klein BP, Lee SY. Developing a lexicon for descriptive analysis of soymilks. J Food Sci 2004;69:259-63.

20. Galán-Soldevilla H, Ruiz Pérez-Cacho P, Jiménez $S$, et al Development of a preliminary sensory lexicon for floral honey. Food Qual Prefer 2005;16:71-7.

21. Noble AC, Arnold RA, Buechsenstein J, et al. Modification of a standardized system of wine aroma terminology. Am J Enol Vitic 1987;38:143-6.

22. Zeppa G, Gambigliani Zoccoli M, Nasi E, et al. Descriptive sensory analysis of Aceto Balsamico Tradizionale di Modena DOP and Aceto Balsamico Tradizionale di Reggio Emilia DOP. J Sci Food Agric 2013;93:3737-42.

23. Talavera-Bianchi M, Chambers DH. Simplified lexicon to describe flavor characteristics of western European cheeses. J Sensory Stud 2008;23:468-84.

24. Etaio I, Albisu M, Ojeda M, et al. Sensory quality control for food certification: a case study on wine. Method development. Food Control 2010;21:533-41.

25. Maitre I, Symoneaux R, Jourjon F, Mehinagic E. Sensory typicality of wines: how scientists have recently dealt with this subject? Food Qual Prefer 2010;21:726-31.

26. Coggins PC, Schilling MW, Kumari S, Gerrard PD. Development of a sensory lexicon for conventional milk yogurt in the United States. J Sensory Stud 2008;23:671-87.

27. European Commission. Commission Regulation (EEC) No. 2568/91 of 11 July 1991 on the characteristics of olive oil and olive-residue oil and on the relevant methods of analysis. In: Official Journal, L 248, 5/9/1991, pp 1-83.

28. European Commission. Commission Regulation (EC) No. 796/2002 of 6 May 2002 amending Regulation (EEC) No 2568/91 on the characteristics of olive oil and olive-pomace oil and on the relevant methods of analysis and the additional notes in the Annex to Council Regulation (EEC) No. 2658/87 on the tariff and statistical nomenclature and on the Common Customs Tariff. In: Official Journal, L 128, 15/5/2002, pp 8-28.

29. European Commission. Commission Regulation (EC) No. 1989/2003 of 6 November 2003 amending Regulation (EEC) No. $2568 / 91$ on the characteristics of olive oil and olive-pomace oil and on the relevant methods of analysis. In: Official Journal, L 295, 13/11/2003, pp 57-77.

30. European Commission. Commission Regulation (EC) No. 640/2008 of 4 July 2008 amending Regulation (EEC) No. 2568/91 on the characteristics of olive oil and olive-residue oil and on the relevant methods of analysis. In: Official Journal, L 178, 5/7/2008, pp 11-16.

31. Gerbi V, Zeppa G, Antonelli A, Carnacini A. Sensory characterization of wine vinegars. Food Qual Pref 1997;8:27-34.

32. Gerbi V, Zeppa G, Beltramo R, Carnacini A, Antonelli A Characterization of white vinegars of different sources with artificial neural networks. J Sci Food Agric 1998;78:417-22.

33. Tesfaye W, Morales ML, Garćia-Parrilla MC, Troncoso AM. Wine vinegar: technology, authenticity and quality evaluation. Trends Food Sci Technol 2002;13:12-21.

34. Morales ML, Benitez B, Tesfaye W, et al. Sensory evaluation of Sherry vinegar: Traditional compared to accelerated aging with oak chips. J Food Sci 2006;71:238-42. 
35. Tesfaye W, Morales ML, Callejón RM, et al. Descriptive sensory analysis of wine vinegar: tasting procedure and reliability of new attributes. J Sensory Stud 2010;25:216-30.

36. IS0 (International Organization for Standardization). ISO 1087:1990. Sensory analysis. Terminology. Geneva: International Organization for Standardization Publications; 1990.

37. ISO (International Organization for Standardization). ISO 5492:1992. Sensory analysis. Vocabulary. Geneva: International Organization for Standardization Publications; 1992.

38. Giboreau A, Dacremont C, Egoroff C, et al. Defining sensory descriptors: towards writing guidelines based on terminology. Food Qual Prefer 2007;18:265-74.

39. ISO (International Organization for Standardization). ISO 4121:1987. Sensory analysis. Methodology. Evaluation of food products by methods using scales. Geneva: International Organization for Standardization Publications; 1987.

40. Meilgaard M, Civillie GV, Carr BT. Sensory evaluation technique, 4th ed. Boca Raton, FL: CRC Press; 1991.

41. ISO (International Organization for Standardization). ISO 8586:1993. Sensory analyses. General guidance for the selection, training and monitoring of assessors. Part 1: Selected assessors. Geneva: International Organization for Standardization
Publications; 1993.

42. ISO (International Organization for Standardization). ISO 8586:1993. Sensory analysis. General guidance for the selection, training and monitoring of assessors. Part 2: Experts. Geneva: International Organization for Standardization Publications; 1993.

43. Frijters JE. Psychophysical models for mixtures of tastants and mixtures of odorants. Ann N Y Acad Sci 1987;510:67-78.

44. Laffort P. Models for describing intensity interactions in odor mixtures: a reappraisal. In: Laing DG, Cain WS, McBride RL, Ache BW. Perception of complex smells and tastes. Sidney: Academic Press; 1989.pp173-88

45. Helson H. Adaptation-level theory. Oxford: Harper \& Row; 1964.

46. Murray JM, Delahunty CM, Baxter IA. Descriptive sensory analysis: past, present and future. Food Res Int 2001;34:461-71.

47. Giudici P. Gluconic acid: genuine criterion of the traditional balsamic vinegar. Industr Bevande 1993;124:123-5.

48. Prescott J, Stevenson RJ. Pungency in food perception and preference. Food Rev Int 1995;11:665-98.

49. Falcone PM, Giudici P. Molecular size and molecular weight distribution impacting traditional balsamic vinegar ageing. J Agric Food Chem 2008;56:7057-66. 